\title{
A UTILIZAÇÃO DA MODELAGEM AMBIENTAL PARASISTEMATIZAÇ̃̃O DO CONHECIMENTO TÁCITO:IDENTIFICAÇÃO DE CORREDORES PREFERENCIAIS PARALINHAS DE TRANSMISSÃO DE ENERGIA ELÉTRICA
}

\author{
Pedro Bueno Rocha Campos*
}

\section{Resumo}

A necessidade de expansão do setor elétrico requer o aprimoramento constante das suas formas de projetar e executar empreendimentos, como linhas de transporte de energia elétrica, sem que se perca nessa evolução o conhecimento já adquirido na experiência consolidada ao longo de tantos anos de crescimento. A possibilidade de se extrair o conhecimento tácito e a experiência através dos padrões de execução, representa oportunidade de estabelecer termos comparativos e parametrizados para à associação com as novas tecnologias e ganho de produtividade para o setor. Os padrões de execução podem ser encontrados na paisagem onde os projetos foram executados. Para tanto, a elaboração de um modelo espacialmente explicito e dinâmico faz-se necessário, visando reconhecer não só o padrão espacial de ocorrência do fenômeno, mas também a sua variação na história. Metodologias robustas, como Pesos de Evidências e LeastCostly Path, auxiliaram está pesquisa a retirar conhecimento sobre o processo de locação de linhas de transmissão de energia da paisagem. Os resultados demostram, além dos locais de preferência para locação destes empreendimentos, a possibilidade de identificação de variáveis não consideras nas tratativas normativas do setor.

Palavras-chave:Linhas de transmissão de energia elétrica. Conhecimento tácito. Pesos de Evidência.

\footnotetext{
*Mestrando em Análise e Modelagem de Sistemas Ambientais. Universidade Federal de Minas Gerais (pbrcampos@gmail.com) 


\section{1-INTRODUÇÃO}

A expansão do sistema de distribuição de energia elétrica requer a construção de novas linhas de transporte e distribuição de energia, são empreendimentos deinfraestrutura básica, necessários ao desenvolvimento de qualquer país e que atendem sem exceção a toda cadeia produtiva e econômica. Quanto mais cresce este setor, mais questões surgem sobre a sua implantação e determinação dos locais de construção destes empreendimentos (EPRI, 2006). Este setor de infraestrutura investe muito no desenvolvimento de melhores tecnologias e sistemas para elaboração e execução destes projetos, são vários os estudos de Pesquisa e Desenvolvimento - P\&D, patrocinados por empresas consolidadas do setor.

Dentre as pesquisas desenvolvidas, destaca-se aqui as relacionadas a escolha elocação dos traçados de linha de transmissão de energia, como a metodologia desenvolvida pelo Electric Power ResearchInstitute- EPRI (2006), o trabalho apresentado por Schmidt (2009) e o P\&D elaborado para a otimização de rotas para linhas de transporte de energia, com o desenvolvimento do software Rota (BELÉM et al, 2009). Todas essas pesquisas tem como base a metodologia multicritério para decisão do traçado e a associação com algoritmos de otimização das rotas, através da análise de custo das alternativas apresentadas.

Concomitante com o desenvolvimento de novas tecnologias, o projeto de uma grande obra de infraestrutura segue uma serie de regras, leis e procedimentos prédefinidosque o acompanham desde sua fase de planejamento até as fases de execução e operação, algo que também é inerente aos projetos de linha transmissão. São varias as normativas relacionadas a este tipo de empreendimento, entre elas temos a NBR 5.422 (1985) e os Procedimentos de Distribuição de Energia Elétrica no Sistema Elétrico Nacional - PRODIST (ANEEL, 2013). Além disso, temos as proposições teóricas sobre elaboração de projetos, como a oferecida por Labegalini et al (1992). Em todas as tratativas descritas existem indicações, proposições e diretrizes gerais sobre a escolha do local onde será implantado o projeto, por exemplo, no PRODIST existe um tópico sobre a escolha e locação de linhas de energia, cuja recomendação se limita a indicar que a alternativa mais viável é a que atenda as normas técnicas de forma a garantir a qualidade e confiabilidade do sistema ao menor custo global, observando-se ainda a legislação ambiental. Os outros documentos encontrados também não dão maiores parâmetros para definição do traçado. 
Entretanto, a real implementação do projeto não está totalmente descrita nesse conjunto de regras e procedimentos, uma vez que, grande parte do conhecimento necessário para tanto pode ser classificado como tácito e inerente a experiência dosprofissionais envolvidos no projeto (RIBEIRO, 2012).

O conhecimento tácito pode ter, entre suas diversas distinções e aprofundamentos, a definição simplista de saber mais do que se consegue falar ou transmitir (POLANYI, 1983). Conforme Ribeiro (2012), a sistematização do conhecimento tácito e da experiência dos profissionais tem oferecido grandes vantagens de produtividade as empresas, cuja experiência foi desenvolvida em longos anos de atuação e com a participação de vários profissionais das mais diversas áreas do conhecimento. Portanto, a sistematização desse tipo de conhecimento tem a complicada tarefa de apreender a forma como foi realizado algo que nem mesmo quem executou tem a habilidade de explicar em termos parametrizáveis ou que não sejam subjetivos ao ponto de variar bruscamente entre diferentes profissionais.

Quando se é utilizado metodologias multicritério na fase de planejamento do projeto parte desse conhecimento tácito pode ser capitalizado e aproveitado, visto que a utilização dessa metodologia possibilita a combinação de vários critérios, atribuindo-se a cada uma dessas variáveis algum peso de ponderação, que por sua vez, são definidos por um conjunto de profissionais que são relacionados à variável ou ao conjunto de variáveis em questão (SADASIVUNI et al, 2009).

A ponderação das variáveis tem como base a inserção de uma escala de preferência ou impacto, positivo ou negativo, para cada variável, sendo 1 menor preferência e 9 maior preferência (SCHMIDT, 2009). Esta escala é preenchida por cada profissional e cada envolvido no desenvolvimento do projeto. Depois cada variável écomparada par a par através de metodologias robustas como o AnalyticalHierarchyProcesss (AHP) que identifica e soluciona problemas de conflitos entre as áreas envolvidas no projeto (NOBREGA, 2009), por exemplo, a dificuldade de viabilizar a otimização de recursos disponíveis, ao mesmo tempo em que se pretende minimizar os impactos ambientais.

Ao ser introduzida uma classificação de preferência, com critérios entre maior e menor, surgem problemas de interpretação sobre o que é maior ou menor para cada profissional (EPRI, 2006). Técnicas são aplicadas para minimizar o desvio entre as classificações e gerar um consenso para a escala aplicável a cada variável. Entretanto,mesmo em consenso, esta escala parte da subjetividade da capacidade de interpretação pessoal. 
Inegável é a relação da capacidade de interpretação com a experiência profissional, mas a possibilidade de se avaliar o que de fato foi executado até então, pelos mesmos profissionais, pode gerar termos comparativos e parametrizados que indiquem melhor qual a real preferência no momento de execução de um projeto de linha de energia. Em outras palavras, a análise do padrão de execução dos projetos de uma mesma empresa ao longo do desenvolvimento de sua experiência pode auxiliar nabusca pelo aperfeiçoamento dos planejamentos e das metodologias de locação dos empreendimentos.

A modelagem dinâmica tem a capacidade de representar a realidade de forma sintética em modelos análogos e abstratos. De acordo com Chistofoletti (1998), a representação em um ambiente controlado do sistema de forças e energias que interagem entre si pode fornecer parâmetros para as variáveis que explicam ou que determinam o funcionamento de determinado fenômeno, como por exemplo, a existência de um acontecimento num lugar específico, ou seja, que obedeça a um padrão espacial.

O estudo sobre a identificação de padrões espaciais tem larga aplicação em pesquisas sobre mudança de uso e cobertura do solo, conforme verificado em Soares- Filho (1998) e Almeida (2003), como também na identificação de padrões para localização de fenômenos, conforme visto em Bonham-Carter (1994) e Nunes (2010).

Portanto, se a existência de linhas de energia for considerada um fenômeno que ocorre na paisagem e determinado por variáveis mensuráveis é possível que os traçados das linhas de transmissão de energia obedeçam a padrões espaciais.

Dessa forma, este artigo propõe uma metodologia de identificação de padrões espaciais na implantação de projetos de obras de infraestrutura de transmissão e transporte de energia elétrica. Foram selecionadas linhas de energia construídas desde a década de 1960 até 2000, buscando padrões espaciais de forma dinâmica, conforme a evolução paisagem.

\section{Materiais e Métodos}

A representação da paisagem considerou as variáveis que tem maior capacidade explicativa para a ocorrência de linhas de energia. Para tanto, foi considerada a normatécnica de especificação de obras desse tipo de empreendimento (NBR 5.422, 1985). Foi utilizada também a tratativa teórica para projetos de linhas de transmissão de energia oferecida por Labegalini et al (1992), bem como as pesquisas desenvolvidas no estudo corredores ótimos para locação das linhas (EPRI, 2006; SCHMIDT, 2009). 
De acordo com Labegalini et al (1992), são várias as soluções possíveis de interligação entre dois pontos de conexão, entretanto são poucas as que podem ser operacionalizadas atendendo aos critérios de viabilidade eletromecânica. A NBR-5.422 (1985) trata que a viabilidade técnica do trajeto da linha deve considerar a tensão nominal, o relevo, o material a ser utilizado (condutores e suportes), a condição de fundação (solo, profundidade e afloramentos), qual o tipo de uso e ocupação dasuperfície para o cálculo da distância do cabo ao solo, a existência de travessias de riose rodovias e a velocidade do vento. Já os estudos desenvolvidos pelo EPRI (2006), trazem inúmeras variáveis, classificadas em grupos, a partir do conjunto de profissionais envolvidos com o projeto e conforme a etapa de desenvolvimento da metodologia proposta.

Basicamente são três etapas, propostas pelo EPRI (2006), de consultas realizadas para proposição da rota mais viável para o projeto. A primeira etapa visa propor um macro corredor preferencial para linhas, utilizando das variáveis: rodovias, declividade (representando o relevo), uso e cobertura solo e linhas de transmissão de energia jáexistentes. A segunda consulta propõe corredores alternativos a partir do macro corredor encontrado e utilizam as variáveis separadas em quatro grupos: $1^{\mathrm{a}}$ variáveis do ambiente antrópico, como proximidade a prédios, densidade de prédios, estrutura fundiária; $2^{\mathrm{a}}$ variáveis do ambiente natural, como áreas alagadas, áreas de mata, parques e áreas de conservação; $3^{\mathrm{a}}$ variáveis técnicas, como linhas existentes, rodovias, gasodutos e relevo; e $4^{\mathrm{a}}$ áreas evitáveis, como cemitérios, sítios arqueológicos, aeroportos e áreas militares. Já a terceira e última etapa realiza a consulta da viabilidade econômica, levantando o custo de cada alternativa encontrada e também faz um refino das alternativas com outras variáveis, como proximidade a edificações industriais ou comerciais. Nesta pesquisa buscou-se identificar padrões espaciais na locação do que o EPRI (2006) denomina como macro corredores preferencias para linhas de energia. Algumas variáveis necessárias para construção de linhas de energia não estão presentes diretamente na paisagem, como o material a ser utilizado na construção, outras variáveis são derivações de variáveis mais gerais, como proximidade ou densidade de ambientes antrópicos. Jávariáveis como condição de fundação e algumas áreas evitáveis não são observáveis diretamente, pelo profissional, quando do planejamento do macro corredor.

Dessa forma, optou-se pela utilização das variáveis: distância a rodovias, distância a linhas existentes, distância a principais rios e cursos de água, uso e cobertura do solo e 
declividade. Compreendem-se estas como o conjunto de variáveis preditoras a maior preferência pela ocorrência de linhas de transporte de energia.

Todas as variáveis foram convertidas para a forma matricial de representação, onde cada célula da matriz tem $60 \times 60$ metros, todas com o mesmo registro geográfico. O uso e ocupação do solo foram extraídos da classificação de imagens Landsat 5, 7 e 8, nos anos de 1975, 1984, 1990 e 2000, visto a disponibilidade de imagens que melhor dividissem o tempo abrangido de análise. Assim, as linhas existentes foram divididas em cinco categorias, conforme o ano de entrada em operação. A primeira classe é das linhas construídas antes de 1975 e as demais classes estão nos intervalos de classificação do uso e cobertura do solo. As demais variáveis foram consideradasestáticas no modelo, ou seja, não possuem variação no tempo.

Para identificação dos padrões espaciais foi utilizada a metodologia proposta por Almeida (2003) e Bonham-Carter (1994). Ambos têm como base o Teorema de Bayesou também denominado Teorema da Probabilidade Condicional, cuja aplicação fornece a probabilidade de ocorrer um fenômeno, no caso a existência de linhas de energia ( $\mathrm{Z}$ ), dado a ocorrência de um conjunto de variáveis explicativas e relacionadas ao fenômeno $(\mathrm{B} \cap \mathrm{C} \cdots \cap$ N), conforme a equação 1:

$$
P(Z \mid B \cap C \ldots \cap N)=P(Z) \frac{P(B \cap C \ldots \cap N \mid Z)}{P(B \cap C \ldots \cap N)}
$$

A equação 1 encontra a probabilidade de ocorrência, mas para saber se há um padrão espacial é necessária a verificação do comportamento das variáveis onde não ocorre $Z$ ( $Z^{\prime}$ ), conforme a equação 2 :

$$
P\left(Z^{\prime} \mid B \cap C \ldots \cap N\right)=P\left(Z^{\prime}\right) \frac{P\left(B \cap C \ldots \cap N \mid Z^{\prime}\right)}{P(B \cap C \ldots \cap N)}=1-P(Z \mid B \cap C \ldots \cap N)
$$

A definição da presença ou ausência de linha de energia estabelece uma variável dependente binaria, com o comportamento de uma função logística quando variada por um conjunto de variáveis explicativas. Como uma função logística tem características não lineares de regressão, há a necessidade da linearização dessa função para que seja possível determinar a 
capacidade ou qual a participação de cada variável explicativa na presença ou ausência da variável dependente (MENDES, 2004). A linearização da função ocorre, de uma forma simplista, quando o intervalo de variação da variável dependente deixa de ser binário, ou seja, variar entre 0 e 1 (MENDES, 2004). A divisão da probabilidade de $\mathrm{P}(\mathrm{Z} \mid \mathrm{B} \cap \mathrm{C} \cdots \cap \mathrm{N})$ por $\mathrm{P}\left(\mathrm{Z}^{\prime} \mid \mathrm{B} \cap \mathrm{C} \cdots \cap \mathrm{N}\right)$ fornece a chance de ocorrência da variável dependente, este conceito é denominado Odds $(\mathrm{O})$ e tende ao $+\infty$ quando a probabilidade de $\mathrm{Z}$ tende a 1 (Equação 3).

$$
O=\frac{P(Z \mid B \cap C \ldots \cap N)}{1-P(Z \mid B \cap C \ldots \cap N)}
$$

Dessa forma, o intervalo de variação passa a ser $0 \leq \mathrm{P}(\mathrm{Z} \mid \mathrm{B} \cap \mathrm{C} \cdots \cap \mathrm{N}) \leq+\infty$ epara eliminar o limite inferior utiliza-se uma transformação logit (logisticprobabilityunit) da Odds, conforme equação 4 :

$$
\text { Logit } O=\ln _{e}\left[\frac{P(Z \mid B \cap C \ldots \cap N)}{1-P(Z \mid B \cap C \ldots \cap N)}\right]=W_{N}^{+}
$$

Onde $W_{N}^{+}$representa o coeficiente do peso de evidência, este termo assume a característica de atribuir um peso de explicação para cada variável independente, onde quanto maior o valor de 0 mais associada é a ocorrência de $Z$ com variável independente em análise. Quando todas as variáveis preditoras são analisadas em conjunto, atribuindo-se a cada uma seu respectivo coeficiente, encontramos o peso de evidência, ou seja, a probabilidade de ocorrência de $\mathrm{Z}$ dado a ocorrência do conjunto $(\mathrm{B} \cap \mathrm{C} \cdots \cap \mathrm{N})$ que representa as variáveis mesuradas na paisagem. Para tanto, convertemos o logit de O novamente para probabilidade e substituímos na equação 1 , conforme as equações 5 e 6 : 


$$
\frac{P(Z \mid B \cap C \ldots \cap N)}{1-P(Z \mid B \cap C \ldots \cap N)}=e^{W_{N}^{+}}
$$

(5)

Simplificando para a equação 1:

$$
P(Z \mid B \cap C \ldots \cap N)=\frac{e^{W_{N}^{+}}}{1+e^{W_{N}^{+}}}
$$

Como a metodologia proposta apenas atribui pesos a variáveis categóricas foi necessário a categorização das variáveis com valores contínuos. A separação dos intervalos das classes categoricas utilizou a metodologia proposta por Agterberg e Bonham Carter (1990) e adaptada por Soares-Filho (2008) no software Dinamica Ego.

Constituídas as variáveis, o modelo faz uma análise dinâmica dos locais de preferência para implantação de linhas de transmissão de energia ao considerar que, dado as faixas de intervalo de análise, as condições para seleção de um corredor no instante t0 são diferentes para o instante $\mathrm{t} 0+\mathrm{t} 1$, ou seja, no instante $\mathrm{t} 0$ o uso e ocupação do solo é diferente do instante t0 + t1, modificando, portanto, a dinâmica da paisagem e, por sua vez, a preferência para implantação de linhas de transmissão de energia. Dessa forma, o modelo interage quatro vezes, uma para cada intervalo de observação, alterando o mapa de uso e ocupação do solo e a distância para as linhas que foram construídas no período anterior. A probabilidade de ocorrência de linhas de energia considera, assim, as linhas que já existiam no período anterior mais as do período em análise, conforme a figura 1. 


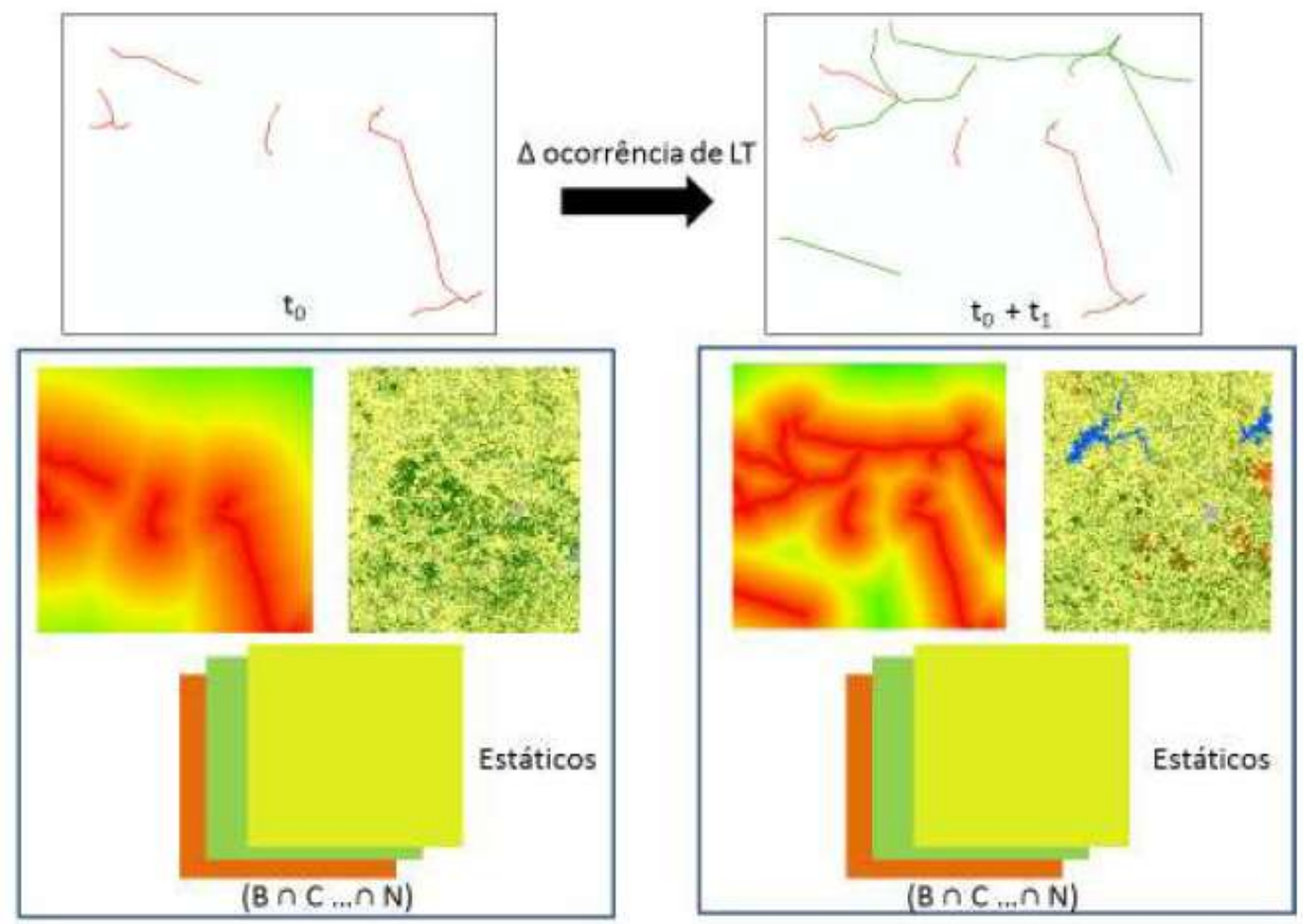

Figura 1:Variação Ocorrência de Linha de Transmissão de Energia em Função da Paisagem

A calibração do modelo para a metodologia utilizada consiste na verificação daindependência entre as variáveis. Para tanto, foram realizados dois testes estatísticos: o cálculo do Coeficiente de Cramer e o Joint InformationUncertainty (BONHAM CARTER, 1994). As variáveis são comparadas par a par e os valores são apresentados para cada comparação. Quanto menor os valores encontrados, menor é dependência entre as variáveis. Bonham Carter (1994) sugere o limite de 0,5 para as duas análises.

O cálculo de um mapa de probabilidade para cada categoria de linha já existentegera, para cada variável, quatro coeficientes de pesos de evidência. Analisando que são quatro paisagens diferentes e que em cada período as linhas de energia foram construídas em locais diferentes, estes coeficientes podem variar para cada período, conforme o local de maior aumento de ocorrência de linhas de energia. Portanto, ao final foi realizada uma medida de tendência para os quatro mapas de probabilidade, através de média simples, visando amortizar coeficientes tendenciosos caso o aumento da ocorrência de linha de energia se desse de forma concentrada em um local especifico. 
A validação do modelo foi feita a partir da locação de um traçado para uma linhaexistente, sendo que esta não foi considerada no modelo. A locação utilizou a metodologia proposta por Soares-Filho et al (2009), que prevê o cálculo de um mapa de custo para sair de qualquer ponto do mapa para um ponto especifico, em outras palavrasé mapa de custo acumulado para um ponto ou local especificado. O algoritmo que faz esse cálculo é do tipo pushbroom e utiliza um mapa que informe os custos de deslocamento de cada célula. O mapa de probabilidade foi considerado como o mapa de custo de cada célula, entretanto é necessário que se utilize no algoritmo o inverso do mapa probabilidade. Com o mapa de custo acumulado calculado, é utilizado um algoritmo denominado LeastCostly Path que encontra o menor custo para atravessar o mapa a partir de um segundo ponto informado.

\section{Aplicação do Modelo}

A aplicação do modelo se deu na região do Triângulo Mineiro em Minas Gerais e parte do estado de Goiás. Os principais municípios abrangidos pela pesquisa foram Uberlândia, Araguari, Uberaba, Ituiutaba e Itumbiara, figura 2. As linhas de transmissão de energia pesquisadas são de responsabilidade da Cemig Distribuição S/A e Cemig Transmissão S/A, as tensões nominais variam entre $69 \mathrm{kV}$ e $500 \mathrm{kV}$. A maioria das linhas de energia tem estruturas metálicas de suporte ao cabeamento e faixas de segurança a partir do eixo central entre 23 metros e 40 metros.

A classificação de uso e ocupação do solo foi separada nas classes: vegetação nativa, área urbana, corpos d'água, silvicultura e atividades agropecuárias. A classificação da classe urbana foi feita de forma manual, pois geralmente as subestações, as quais as linhas de energia se conectam, são construídas próximas as cidades e com a expansão dos limites da área urbana forma-se corredores dentro da área urbana sem que seja essa a ocupação do corredor. Como um exemplo segue a figura 2, onde a linha foi construída em 1998 e até 2007 não existia a utilização urbana no local, já em 2013 um condomínio vertical foi construído no entorno e o corredor é agora umcanteiro central de uma larga avenida. 


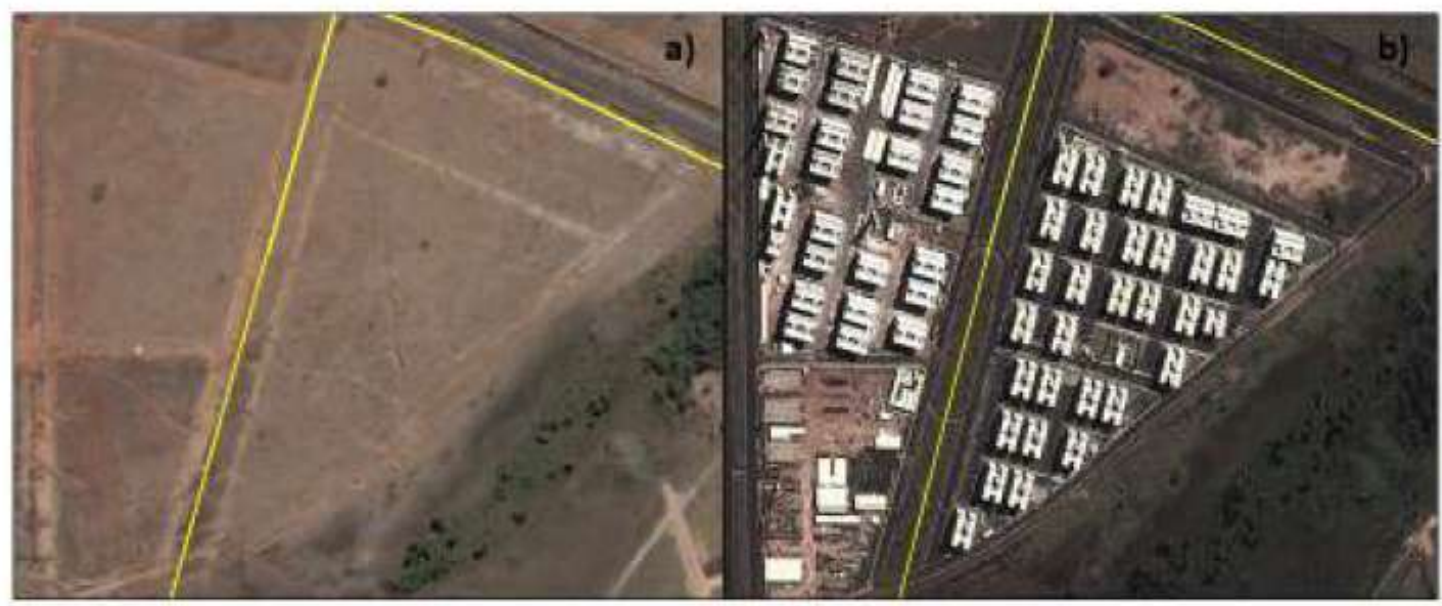

Fonte: Google Earth, 2013.

Figura 2: Corredor de LT em Área Urbana. Figura a) ocupação em 2007 e figura b) em 2013

Após a classificação de uso e ocupação do solo, as variáveis contínuas foram categorizadas e o modelo foi calibrado a partir dos testes estatísticos propostos na metodologia. Todas as variáveis foram consideradas independentes, em todos os intervalos de análise, conforme as tabelas 1, 2, 3 e 4 abaixo:

Tabela 1: Testes Estatísticos de Independência das Variáveis. Antes de 1975 a 1975

\begin{tabular}{cccc}
\hline \multicolumn{4}{c}{ Intervalo: antes de 1975 a 1975 } \\
\hline Primeira Variável & Segunda Variável & Crammer & Joint_Uncertainty \\
\hline d_linhas & d_rios & 0 & 0,016218961 \\
\hline d_linhas & d_rodovias & 0,066194305 & 0,04377679 \\
\hline d_linhas & declividade & 0,049206133 & 0,008218775 \\
\hline d_linhas & uso do solo & 0,099596345 & 0,003878738 \\
\hline d_rios & d_rodovias & 0,018687253 & 0,007593339 \\
d_rios & uso do solo & 0,018839911 & 0,001396424 \\
d_rios & uso do solo & 0,0854748 & 0,003050208 \\
d_rodovias & declividade & 0,020017316 & 0,001491998 \\
d_rodovias & uso do solo & 0,075351456 & 0,002415976 \\
declividade & uso do solo & 0,058527886 & 0,00382444 \\
\hline
\end{tabular}


Tabela 2: Testes Estatísticos de Independência das Variáveis. 1975 a 1984

\begin{tabular}{cccc}
\hline & \multicolumn{2}{c}{ Intervalo: 1975 a 1984 } & \\
\hline Primeira Variável & Segunda Variável & Crammer & Joint_Uncertainty \\
\hline d_linhas & d_rios & 0 & 0,017170509 \\
\hline d_linhas & d_rodovias & 0,051508186 & 0,031611874 \\
\hline d_linhas & declividade & 0,034849175 & 0,00462175 \\
\hline d_linhas & uso do solo & 0,090457994 & 0,004862327 \\
\hline d_rios & d_rodovias & 0,01883212 & 0,007633734 \\
d_rios & uso do solo & 0,018900157 & 0,001399669 \\
d_rios & uso do solo & 0,128724494 & 0,009439486 \\
d_rodovias & declividade & 0,020077545 & 0,001492912 \\
d_rodovias & uso do solo & 0,110210641 & 0,006386259 \\
declividade & uso do solo & 0,104559773 & 0,014072607 \\
\hline
\end{tabular}

Tabela 3: Testes Estatísticos de Independência das Variáveis. 1984 a 1990

\begin{tabular}{cccc}
\hline & \multicolumn{2}{c}{ Intervalo: 1984 a 1990 } & \\
\hline Primeira Variável & Segunda Variável & Crammer & Joint_Uncertainty \\
\hline d_linhas & d_rios & 0 & 0,017371562 \\
\hline d_linhas & d_rodovias & 0,053103078 & 0,031725774 \\
\hline d_linhas & declividade & 0,035036143 & 0,004559375 \\
\hline d_linhas & uso do solo & 0,09796791 & 0,005333947 \\
\hline d_rios & d_rodovias & 0,01894504 & 0,007623664 \\
\hline d_rios & uso do solo & 0,018897789 & 0,00140096 \\
d_rios & uso do solo & 0,135973692 & 0,010204318 \\
d_rodovias & declividade & 0,020027041 & 0,001492944 \\
\hline d_rodovias & uso do solo & 0,113629722 & 0,00655491 \\
\hline declividade & uso do solo & 0,143059371 & 0,025577679 \\
\hline
\end{tabular}


Tabela 4: Testes Estatísticos de Independência das Variáveis. 1990 a 2000

\begin{tabular}{cccc}
\hline \multicolumn{4}{c}{ Intervalo: 1990 a 2000 } \\
\hline Primeira Variável & Segunda Variável & Crammer & Joint_Uncertainty \\
\hline d_linhas & d_rios & 0 & 0,015473675 \\
\hline d_linhas & d_rodovias & 0,056175843 & 0,038585878 \\
\hline d_linhas & declividade & 0,03500676 & 0,00452274 \\
\hline d_linhas & uso do solo & 0,10133249 & 0,005624459 \\
\hline d_rios & d_rodovias & 0,018696047 & 0,007645479 \\
\hline d_rios & uso do solo & 0,018894407 & 0,001399417 \\
\hline d_rios & uso do solo & 0,141068068 & 0,010335482 \\
\hline d_rodovias & & & \\
\hline d_rodovias & declividade & 0,020109108 & 0,00149431 \\
\hline declividade & uso do solo & 0,101630207 & 0,005669526 \\
\hline & uso do solo & 0,165210011 & 0,034161814 \\
\hline
\end{tabular}

Foram calculados quatro coeficientes para cada categoria das variáveis. Afunção de variação dos coeficientes foi analisada em relação a cada variável explicativa. Os coeficientes que não descreviam nenhuma função ou apresentavam baixa correlação com as variáveis explicativas foram desconsiderados, conforme os gráficos abaixo:

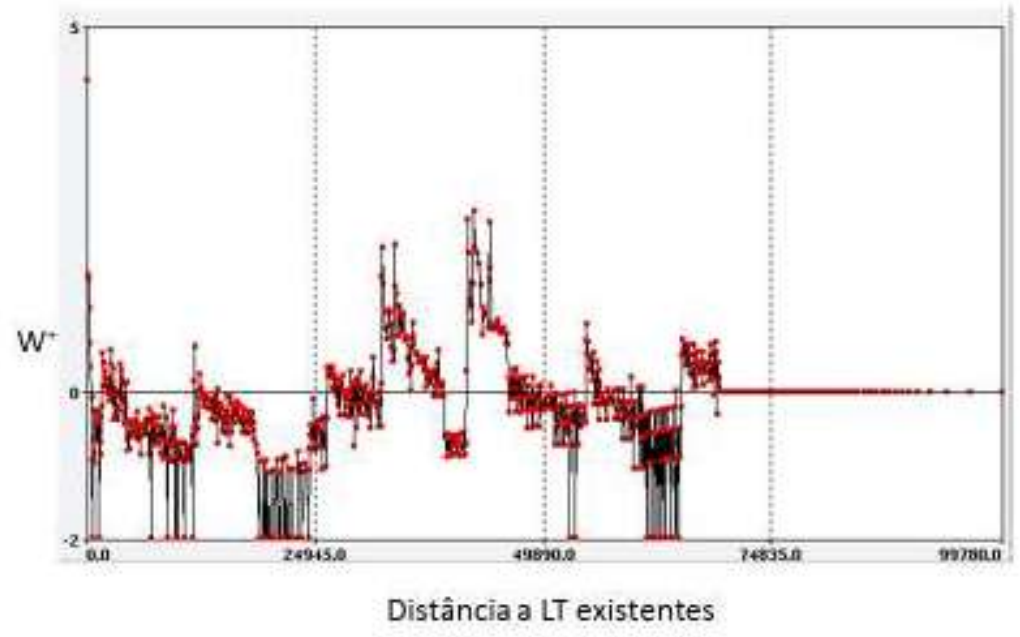

Gráfico 1: Função de W+ em relação à distância de LT existentes

No caso do gráfico 1 então consideradas todas as classes de distância a linhas de energia existentes, chegando a quase $100 \mathrm{~km}$ de análise. Não há uma função bem descrita, pois após uma distância está variável perde a capacidade explicativa. Já no gráfico 2 vemos uma função de 
variação para distâncias até $3 \mathrm{~km}$, onde quanto mais próximo a linha maior é a preferência pela sua ocorrência.

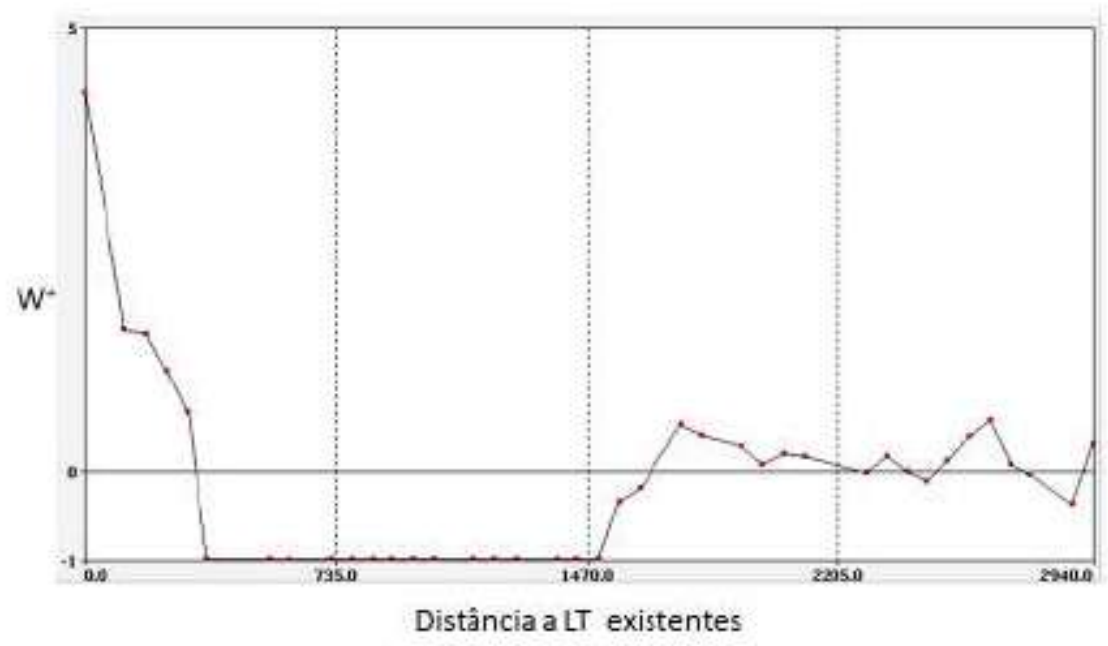

Gráfico 2: Função ajustada de $\mathrm{W}+$ em relação à distância de LT existentes

Após o ajuste dos coeficientes os mapas de probabilidade, ou melhor, de pesos de evidencia são calculados e a média dos quatro períodos indica o mapa final de preferência para locais de implantação de linhas de transporte de energia. A figura 3 é o mapa final de pesos de evidência com os coeficientes ajustados, as cores quentes indicam maior probabilidade e as frias menor probabilidade de ocorrência. 


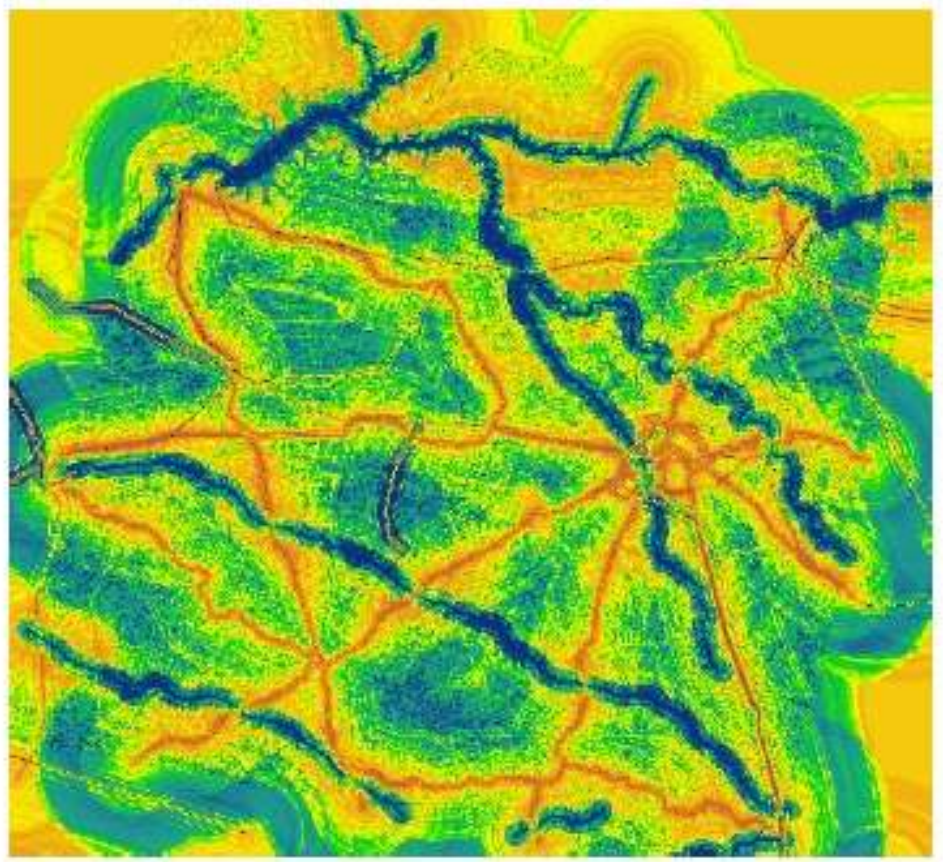

Figura 3: Mapa de Probabilidade com $W_{N}^{+}$os Ajustados

Os locais de maior probabilidade no caso estudado representam a proximidade com linhas de energia e rodovias existentes. Enquanto que as menores probabilidades indicam a proximidade a rios e áreas urbanas. Como a região não tem muita variação de declividade as funções encontradas para os coeficientes dessa variável explicativa não têm tanta representatividade quando comparada com as outras. As figuras 4 a) e 4 b) mostram a comparação entre o mapa de probabilidade calculado com os coeficientes ajustados e sem o ajuste:
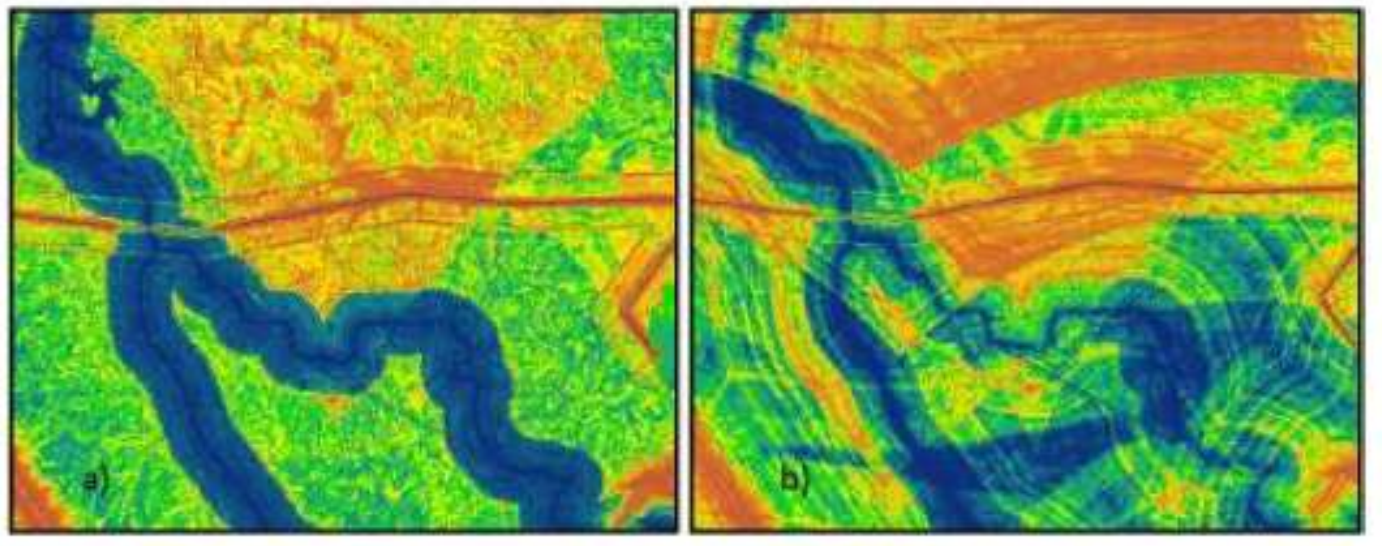

Figura 4: Comparação dos Mapas de Probabilidade. Figura a) com os $W_{N}^{+}$ajustados figura b) sem ajuste 
É possível notar os ruídos da falta de correlação na figura 4b). O ajusteestabelece, além de uma melhor correlação, o melhor funcionamento de todas as variáveis, sem que ocorra o enviesamento de nenhuma variável.

Caso o mapa de pesos de evidência não fosse ajustado, ao se procurar uma rotapreferencial seria traçada uma rota com muitos vértices, considerando que coeficientes enviesados pela falta de função atrairiam ou repeliriam com mais intensidade que os coeficientes que obedecem a função.

O mapa de probabilidade foi validado ao ser traçada uma rota para uma linhaexistente que não foi considera no modelo. A figura 5 mostra em azul a linha traçada pelo modelo e em vermelho a linha existente que não foi considerada, as linhas em verde são existentes e foram utilizadas no modelo: 


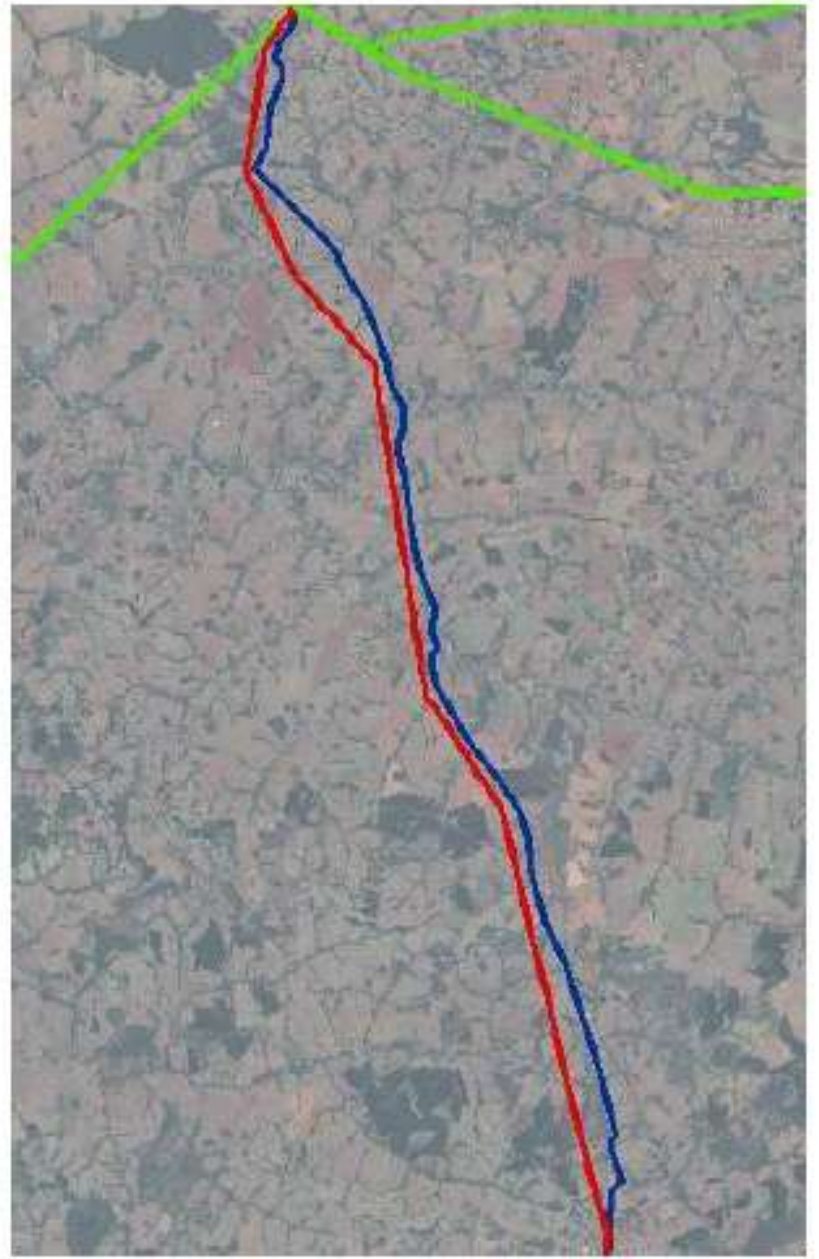

Figura 5: Comparação da Rota Traçada pelo Modelo com a Linha Existente

O comprimento da linha existente é de $63 \mathrm{~km}$ e da linha traçada pelo modelo tem64,4 $\mathrm{km}$ um aumento de 1,4 km. Nota-se, entretanto, a existência de mais vértices na linha modelada, quando comparada com a existente. Fato esse que pode ser justificado pelo ajuste necessário ao projeto executivo e dado quantidade de variáveis explicativas utilizadas no modelo.

A figura 6 mostra um ponto de desvio da linha modelada em função do mapa de probabilidade: 


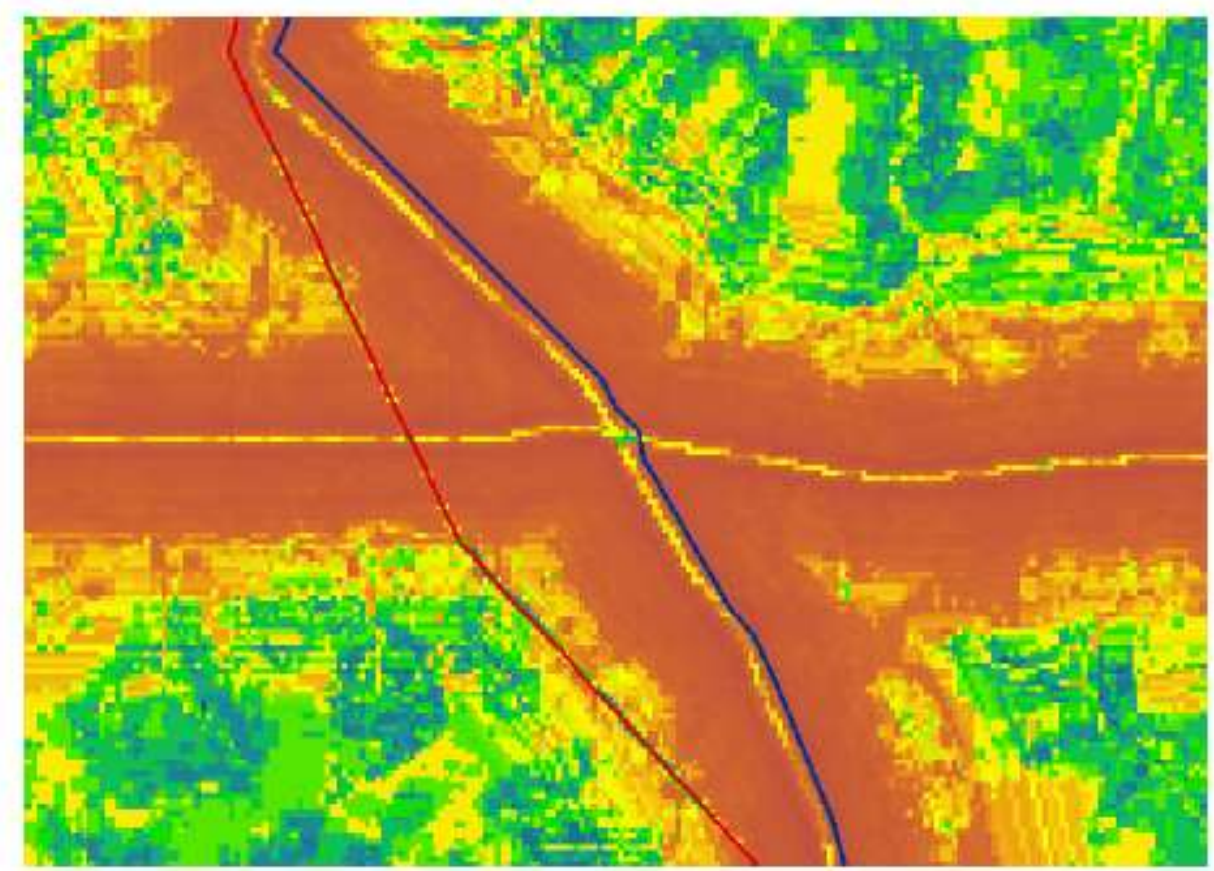

Figura 6: Desvio da Linha Modelada

\section{Considerações Finais}

Do objetivo proposto inicialmente, é possível concluir, nesta fase da pesquisa,que existe a possibilidade de se extrair um padrão de execução de projetos, tomando-se como ponto de referência projetos já executados. No estudo de caso, foi possível perceber a preferência para locação de linhas de transporte de energia elétrica quando associada a experiência de mais de vinte e cinco anos de projetos de uma mesmaempresa. Os termos de preferência foram parametrizados e validados, sendo, portanto, passíveis de replicação para projetos não existentes.

Os fatos que comprovam tal afirmativa são observáveis quando foi demonstrada a função de regressão, cuja variável dependente é a probabilidade de ocorrência de linhade energia dado os locais onde ela já ocorre. Além disso, todas as variáveis preditorasforam consideras independentes entre si e, quando ocorre a existência de linhas de energia, os coeficientes tem uma função correlacionada com a variação das variáveis independentes.

As diferenças encontradas no teste de validação evidenciam que o projeto executivo tem um refino na locação que a escala abordada nesse estudo não abrange. Por outro lado, as diferencias demonstram outro ponto importante que é a possibilidade de identificação de outras variáveis explicativas ao modelo e a possibilidade de retirar do modelo conhecimentos não abordados nas tratativas regulatórias. 
Propõem-se em continuidade a pesquisa a aplicação do modelo em outras regiões, com características diferentes da estudada. Dessa forma será possível estabelecer se há um determinismo para as variáveis utilizadas ou se existe anecessidade de calibração do modelo para cada tipo de região. Outra consideração necessária, é a possibilidade dos parâmetros levantados por este estudo serem utilizados em pesquisas de otimização e desenvolvimento de novas tecnologias aplicadas a locação e projetos de linhas de transporte de energia.

\section{Referências}

ABNT. Projeto de Linhas Aéreas de Transmissão de Energia Elétrica (NBR 5.422).Rio de Janeiro: ABNT, 1985.

AGTERBERG, F.P; BONHAM-CARTER, G.F. Derivingweightsofevidencefromgeosciencescontourmaps for thepredictionofdiscreteevents. XXII Int. Symposium AP-COM, 1990. 381-395

ALMEIDA, C. M. et al. "Stochasticcellularautomatamodelingofurbanland use dynamics: empiricaldevelopmentandestimation" . Computers, EnvironmentandUrban Systems, New York, v. 27, n. 5, p. 481-509, Sept. 2003

BELEM, F. L.; FONSECA, A. R.; PIMENTA L.C.A; REZANDE D.T; TAVARES T.H.B.C; MESQUITA R.C . Ferramenta para seleção de corredor de linha aérea de transmissão utilizando geoprocessamento. In: XIV Simpósio Brasileiro de Sensoriamento Remoto, 2009, Natal. Anais dos XIV SBSR - Simpósio Brasileiro de Sensoriamento Remoto. São Jose dos Campos: Ed Inpe, 2009. v. 1. p. 3559-3566.

BELEM, F. L.; FONSECA, A. R.; Tavares T.H.B.C; Rocha F.T ; MESQUITA R.C; PIMENTA L.C.A . ROTA. 2009.

BONHAM-CARTER, G. F..Geographicinformation systems for geoscientistsmodellingwith GIS. New York: Pergamon. 1994.

CHORLEY, R.J; HAGGET, P. Modelos Integrados em Geografia. Rio de Janeiro, EditoraLivros Técnicos e Científicos S.A., 1974. 279 p.

CHRISTOFOLETTI, A. Modelagem de Sistemas Ambientais. São Paulo: Edgar Blücher, 1998. $236 \mathrm{p}$.

LABEGAlini, P. R., LABEGALINI, J. A., FUCHS, R. D. and ALMEIDA, M. T. (1992). Projetos Mecânicos das Linhas Aéreas de Transmissão, 2a. edn, Edgard Blücher.

NOBREGA, R. 2009. UnderstandingSpatial-Criteriadecisionmaking: Na analyticaldemonstrationof AHP-based MCDM andhow it isused in GIS. In National Consortium for Remote Sensing in Transportation (NCRST) AnnualWorkshop. Memphis: NCRST/GeosystemsResearchInstitute. http://www.gri.msstate.edu/publications/docs/2009/05/5939MCDM1_undestanding\%20 AHP.pdf. 
NUNES, F.S.M. Valoração florestal através de modelagem da rentabilidade da extração de castanha-do-brasil em Madre de Dios, Peru. Brasil. Thesis (Msc). Mestrado em Análise e Modelagem de Sistemas Ambientais. Universidade Federal de Minas Gerais. 2010. Polanyi, M. (1983 [1966]). The tacitdimension. London: Routledge\&Kegan Paul.

RIBEIRO, R. (2007a). KnowledgeTransfer. Unpublisheddoctoraldissertation,Cardiff University, Schoolof Social Sciences, Cardiff, UK. Procedimentos de Distribuição de Energia Elétrica no Sistema Elétrico Nacional -PRODIST, Agência Nacional de Energia Elétrica ANEEL, 2013. URLhttp://www.aneel.gov.br/area.cfm?idArea $=82$

SADASIVUNI, R., C. G. O' HARA, R. A. A. NOBREGA, and J. DUMAS. 2009. A transportationcorridor case study for Multi-Criteriadecisionanalysis. In Proceedings: American SocietyofPhotogrammetryand Remote SensingAnnualConference. Baltimore, MD. URL: www.asprs.org/a/publications/proceedings/baltimore09/0082.pdf.

SCHMIDT, A. J. 2009. Implementing a GIS Methodology for Siting High VoltageElectric TransmissionLines. Volume 11, Papers in ResourceAnalysis. 17 pp. Saint Mary'sUniversityof Minnesota University Central Services Press

SOARES-FILHO, B.S; RODRIGUES, H.O; FALIERI, A; COSTA, W.L., Dinâmica EGO. 2008. Tutorial http://www.csr.ufmg.br/dinamica

STICH, B.; HOLLAND, J. H.; NOBREGA, R. A. A.; O'HARA, C. G. Usingmulticriteriadecisionmakingtohighlightstakeholdersvalues in thecorridorplanningprocess. JournalofTransportand Land Use, v. 4, p. 105-118, 2011 